\title{
Colonias populares consolidadas del área metropolitana de Guadalajara. Retos y oportunidades
}

\author{
Edith R. Jiménez Huerta \\ Heriberto Cruz Solís
}

Universidad de Guadalajara, 2015

\section{Myriam Guadalupe Colmenares López*}

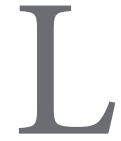

a obra se deriva de una investigación conjunta realizada en once ciudades de nueve países: Monterrey, Ciudad de México y Guadalajara, en México; Buenos Aires, Argentina; Bogotá, Colombia; Lima, Perú; Santo Domingo, República Dominicana; Montevideo, Uruguay; Recife, Brasil; Santiago, Chile, y Guatemala, Guatemala. Esta investigación se suma a los esfuerzos de la Red Latinoamericana de Vivienda (Latin American Housing Network) por estudiar los asentamientos consolidados de la población de bajos ingresos.

Algunos aspectos de la parte metodológica son: la elaboración de una base de datos actualizada de los asentamientos irregulares en cuatro municipios del área metropolitana de Guadalajara: Guadalajara, Zapopan, Tlaquepaque y Tonalá. En total fueron 460 asentamientos detectados cuyas fuentes de información provienen de la Comisión para la Regularización de la Tenencia de la Tierra (Corett), el Programa de Certificación de Derechos Ejidales y Titulación de Solares Urbanos (Procede), la Secretaría de Desarrollo Urbano (Sedeur) y la Procuraduría de Desarrollo Urbano (Prodeur). Los datos fueron ratificados por medición cartográfica y recorridos de campo, dada la discrepancia en la información proporcionada por cada una de las instituciones.

Las colonias irregulares incluidas son aquellas cuyo origen es posterior a 1985, por ser un periodo de acelerada urbanización en Guadalajara, además de considerar que veinte años (de 1985 a 2005, periodo propuesto para el estudio) es tiempo suficiente para la consolidación física de una colonia popular. Entre las colonias

* Profesora-investigadora del Centro Universitario de los Valles, Universidad de Guadalajara, myriamc@valles.udg.mx 
elegidas para el estudio, dado su origen ejidal, se encuentran la Echeverría y Rancho Nuevo de Guadalajara y la Jalisco de Tonalá. Se aplicaron 300 cuestionarios y se hicieron nueve entrevistas a profundidad, que permitieron construir el árbol genealógico de los habitantes, así como reconstruir su historia de vida y las modificaciones realizadas a la vivienda.

El objetivo del trabajo, organizado en siete capítulos, es caracterizar el complejo proceso de consolidación de los asentamientos, la transformación física de las viviendas, la situación jurídica de la propiedad y los cambios en las condiciones sociales de sus habitantes.

El libro comienza explicando los términos "asentamiento regular", "colonia popular", "proceso de consolidación" y "tenencia de la tierra". El estado de la cuestión parte del uso y la definición de un asentamiento humano a partir del llamado slum (que surge en Inglaterra y es adoptado por la Organización de las Naciones Unidas) hasta concretarlo y adaptarlo a casos específicos de América Latina, donde se habla de urbanización popular, producción social del espacio o simplemente del hábitat. Sin embargo, los autores apuestan por la expresión "colonias populares" para definir los asentamientos que presentan irregularidades (jurídicas) en la tenencia de la tierra al momento de su adquisición u ocupación y que fueron autoproducidos paulatinamente.

Asimismo, el primer apartado explica la metodología utilizada y algunos conceptos de relevancia como "tenencia de la tierra", "tenencia residencial" y el contexto del tema en las políticas públicas, que son abordados en capítulos siguientes.

El segundo capítulo presenta un análisis histórico de la conformación del desarrollo urbano de la zona metropolitana de Guadalajara a partir de su dinámica espacial y del crecimiento de la población. Nos lleva de la mano desde la primera conurbación en Guadalajara en el siglo XVIII, la expansión de la ciudad y el surgimiento de las modernas colonias residenciales en tiempos de Porfirio Díaz hasta describir los acontecimientos que vivió como ciudad en la recesión económica, el gigantismo urbano y los primeros intentos legales de ordenamiento urbano de Guadalajara en el siglo XX. Describe el inicio de la metropolización de Guadalajara en los años setenta del siglo pasado y la aparición de asentamientos irregulares en la periferia por la creciente inmigración rural de personas de bajos ingresos en busca de mejorar su calidad vida. Este fenómeno presenta similitudes con el patrón de transformación de otras ciudades de América Latina en donde la ciudad central tiende a decrecer al mismo tiempo que crecen la periferia y el número de asentamientos irregulares en ella.

El tercer capítulo, dividido en tres partes, analiza las características generales de las colonias populares y de sus habitantes. Comienza con una revisión bibliográfica 
para definir al "asentamiento de origen informal o irregular" y situarlo en algún punto de un continuum entre la informalidad y la formalidad dada la diversidad de características para cada caso de estudio. Utiliza el término de "colonias populares consolidadas" para referirse a las urbanizaciones populares que tienen más de veinte años de antigüedad y que en algún momento de su conformación fueron asentamientos irregulares, además de estar asociadas al grado de estándar normativo, urbano, social y de calidad de vida. Como segunda parte, se realiza una caracterización del "anillo" de colonias populares consolidadas, en donde se realza a aquellas con más de veinte años de antigüedad en cuanto a su dimensión, ubicación, densidad, servicios, tipos de propiedad involucrados y situación de regularización; comienza, así, el análisis a partir del primer contorno de crecimiento suburbano de la época moderna, situado entre 1961 y 1985 y denominado "anillo de colonias populares consolidadas".

Para cerrar el capítulo, se describe el panorama socioeconómico de la población residente, que presenta condiciones sociales y económicas menos favorables que la población del resto del área metropolitana. Resalta la importancia de incorporar e involucrar a la población de las colonias populares en proyectos dentro y fuera de la vivienda, ya que pasadas las dos décadas persisten las condiciones socioeconómicas insatisfactorias para los habitantes de ellas.

En el cuarto capítulo se analiza el contexto físico y social que presentan las tres colonias en los niveles macro o regional, meso o colonia y micro o relación ciudadano-entorno inmediato, con la intención de generar políticas públicas que mejoren su situación. Del análisis regional o macro se deriva que el proceso de urbanización de las colonias se vio favorecido por la ubicación, superficie, vialidades e íconos representativos de las colonias. A nivel de colonia o meso, el uso de suelo que predomina es el habitacional, comercial, comercial habitacional e industrial, y se carece de espacios recreativos. Esta diversidad de usos de suelo significa que no existió un control sobre los mismos y se diversificaron las actividades sociales y económicas en el interior de las colonias. Por último, en el nivel micro los autores mencionan que los apoyos recibidos para equipar de infraestructura a las colonias son desiguales y dependen del municipio al que pertenecen; sin embargo, las tres colonias coinciden en que prevalecen las carencias en infraestructura, a la par que presentan diversos problemas sociales, como delincuencia y drogadicción.

El quinto capítulo analiza el proceso de transformación de los lotes, las viviendas y las familias en colonias populares. De la mayoría de los entrevistados resalta la compra del terreno sin edificar, lo cual da opción a iniciar la vivienda a su gusto y de acuerdo con sus posibilidades económicas. Solo aquellos lotes con construcción y vivienda ofrecen otras posibilidades de alojamiento a familiares cercanos o a público 
en general, con la opción de arrendar la vivienda. En el caso del alojamiento familiar, la construcción puede ser vertical (de dos o tres niveles) o bien la que hace eficientes los espacios de la vivienda (familias que habitan en un cuarto) conforme se incrementa la familia. En tanto, las viviendas en alquiler son una opción para aquellas familias de escasos recursos que sacan provecho a su vivienda, en renta ya sea para vivienda o para negocio. En esta primera etapa de consolidación de las colonias se incrementa la superficie construida de las viviendas, la densificación de los lotes que albergan no solo a la familia nuclear (de cuatro a cinco miembros) sino también a futuras generaciones (la pareja fundadora, sus hijos y sus nietos).

Asimismo, se señalan los problemas físicos que las viviendas han sufrido después de veinte años de estar en servicio y que han pasado por procesos de autoconstrucción a medida que cambian las necesidades de sus habitantes. Como resultado de ello, se identificaron problemas relacionados en mayor medida con la evolución física o construcción de la vivienda (aparición de goteras y humedad en techos o paredes), y en menor medida con el funcionamiento de la misma (falta de energía eléctrica y drenaje). Adicionalmente, las viviendas tuvieron que ser transformadas físicamente al instalar protecciones en puertas y ventanas ante la presencia de problemas sociales como la violencia y el narcotráfico. Por último, este capítulo analiza las cuestiones de sucesión, testamento y herencia; se encuentra que existe poco interés por arreglar la situación jurídica de la vivienda.

El sexto capítulo presenta una revisión de los programas de lucha contra la pobreza que tienen incidencia en los asentamientos irregulares ya consolidados. Las categorías que relacionan la pobreza con los asentamientos irregulares en México son muy diversas en las últimas décadas. Ningún programa de apoyo instrumentado por el gobierno ha logrado abatir las deficiencias en materia de apoyo para la adquisición, transformación o mantenimiento legal de las viviendas. Del capítulo se concluye que aún falta mucho por hacer en materia de política pública urbana que evite que trasciendan los problemas que enfrentaron las primeras generaciones y que se acentúan para la segunda y tercera generaciones.

A manera de conclusión, los autores encontraron que los asentamientos de origen irregular consolidados se caracterizan por pertenecer a los dueños originales de la propiedad; algunos han conservado su propiedad por veinte o cuarenta años. La existencia de movilidad residencial entre los habitantes de las colonias populares es mayor a partir de la segunda y tercera generaciones; son los de esta última los menos favorecidos por las políticas de vivienda del gobierno. De igual manera, un hallazgo importante derivado de esta investigación es la certeza jurídica de los predios: el $95 \%$ de los dueños tiene escriturada su propiedad. Sin embargo, se requiere fomentar la cultura de sucesión y herencia en vida para evitar conflictos futuros por la tenencia de la propiedad. 
En general, es una obra recomendada para el lector que gusta de reflexionar sobre los fenómenos sociales, y en particular sobre el fenómeno de la vivienda y su situación jurídica en los llamados "asentamientos irregulares". Ahora los conocemos gracias a la preocupación de estos investigadores por contestar interrogantes de la vida, lo que nos permite saber la dinámica de las familias en las colonias consolidadas. 